\title{
Use of G-protein fusions to monitor integral membrane protein-protein interactions in yeast
}

\author{
Kathleen N. Ehrhard'1, J örg J . J acoby², Xin-Yuan Fu², Reinhard J ahn'1,3, and Henrik G. Dohlman* \\ Departments of ${ }^{1}$ Pharmacology and ${ }^{2}$ Pathology, Yale University School of M edicine, N ew H aven, CT 06536. 3Present address: Department of N eurobiology, \\ M ax-Planck-Institute for Biophysical Chemistry, Am Fassberg, D-37077 Goettingen, Germany. *Corresponding author: (henrik.dohlman@yale.edu).
}

\begin{abstract}
The control of protein-protein interactions is a fundamental aspect of cell regulation. Here we describe a new approach to detect the interaction of two proteins in vivo. By this method, one binding partner is an integral membrane protein whereas the other is soluble but fused to a G-protein $\gamma$-subunit. If the binding partners interact, G-protein signaling is disrupted. We demonstrate interaction between known binding partners, syntaxin 1a with neuronal Sec 1 (nSec1), and the fibroblast-derived growth factor receptor 3 (FGFR3) with SNT-1. In addition, we describe a genetic screen to identify nSec 1 mutants that are expressed normally, but are no longer able to bind to syntaxin 1a. This provides a convenient method to study interactions of integral membrane proteins, a class of molecules that has been difficult to study by existing biochemical or genetic methods.
\end{abstract}

Received 25 M ay 2000; accepted 1 August 2000

All biological processes require precise control of protein activity. Proteins can be regulated by post-translational modifications, altered localization, or association with regulatory subunits or other components of a supramolecular structure (e.g., ribosome, cytoskeleton).

In the past, protein-protein interactions have typically been studied using biochemical techniques such as cross-linking, coimmunoprecipitation, and co-fractionation by chromatography. A disadvantage of these techniques is that interacting proteins often exist in low abundance and are therefore difficult to detect. M oreover, once an interaction is detected, the newly identified protein still must be isolated and sequenced, before the gene can be identified. Another disadvantage is that these methods do not immediately provide information about which domains of a protein are involved in the interaction.

To address technical difficulties associated with the biochemical characterization of protein-protein interactions, alternative genetic methods have been developed. One such method is the yeast twohybrid system, wherein two proteins are fused to either the DNAbinding domain or the transcription-activation domain of Gal4 (refs 1,2). If the two proteins interact, the function of Gal4 is reconstituted (Fig. 1). Transcriptional activation can be detected using the appropriate promoter and a reporter gene, such as lacZ (encodes $\beta$-galactosidase). This approach allows the rapid detection of protein binding partners, including the relevant interacting domains, and immediately provides the gene that encodes the identified interacting proteins. Various permutations of the two-hybrid method have been described, including the split-ubiquitin system ${ }^{3,4}$, the SO S-recruitment system ${ }^{5,6}$, dihydrofolate reductase complementa$\operatorname{tion}^{7,8}$, and $\beta$-galactosidase complementation ${ }^{9,10}$.

Although the yeast two-hybrid system has greatly facilitated the study of protein-protein interactions, there are some situations where it is not suitable. For instance, the method relies on the interaction of two proteins in the nucleus of the cell, so the method is not useful for the study of most integral membrane proteins. M oreover, if one of the proteins is a transcriptional activator, it may itself induce transcription of the reporter gene. Finally, the yeast twohybrid system requires that both proteins be expressed as fusion proteins, resulting in the possible loss of function.

Here we describe a method to monitor protein-protein interaction, using the well-characterized G-protein signaling pathway as the readout. G-protein-coupled receptors can respond to hormones, neurotransmitters, odors, and light. Receptor activation triggers a conformational change in the G-protein $\alpha$-subunit, exchange of GDP for GTP, and dissociation of $G \alpha$ from the G-protein $\beta \gamma$-subunits. Depending on the system, either $\mathrm{G} \alpha$ or $\mathrm{G} \beta \gamma$ can activate downstream effectors, until GTP is hydrolyzed and the protein reverts to the inactive conformation (Fig. 1). In yeast, pheromone stimulation leads to activation of a $G$ protein composed of the products of the GPA1 $(G \alpha)$, STE4 $(G \beta)$, and STE18 $(G \gamma)$ genes. G $\beta \gamma$ in turn activates a kinase signaling cascade that culminates in growth arrest, new genetranscription, cell fusion, and mating ${ }^{11}$.

In the method described here, two protein-binding partners have been tested: syntaxin 1a with nSecl, and FGFR3 with SNT-1. Syntaxin la was chosen because it has a well-characterized function in synaptic vesicle fusion, and it is a proven drug target. nSecl binding prevents syntaxin la assembly with the "SNARE core complex," which is needed for bilayer fusion. An inhibitor of syntaxin is used therapeutically to treat muscle spasms and spasticity that result from inappropriate neurotransmitter release ${ }^{12,13}$. FGFR3 was selected becauseit plays a key role in cell differentiation and proliferation, and mutations of the receptor are associated with birth defects and cancer ${ }^{14,15}$. Binding of fibroblast growth factor to FGFR3 leads to receptor dimerization, tyrosine phosphorylation (including receptor autophosphorylation), and the recruitment of cytoplasmic signaling proteins that transmit the signal. Other cytoplasmic proteins, such as SNT-1, bind permanently to the intracellular domain of the receptor ${ }^{16,17}$.

The ability to monitor the activity of these proteins expressed in their native form (not as a hybrid) and localized at the cell membrane (not in the nucleus) should prove useful in screening for mutants, drugs, or other proteins that alter their binding properties. 


\section{RESEARCH ARTICLES}

A

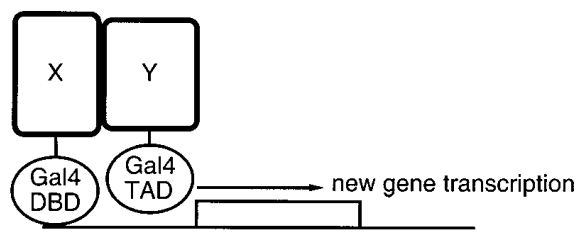

B

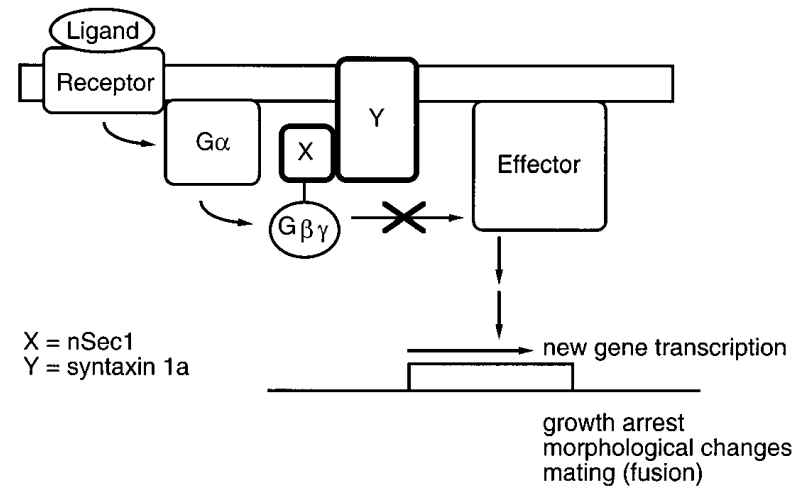

Figure 1. Use of G-protein fusions to monitor integral membrane protein-protein interactions in yeast. (A) In the traditional two-hybrid method the interaction between protein $X$ and protein $Y$ occurs in the nucleus. Reconstitution of the Gal4 DNA-binding domain (DBD) and transcription-activation domain (TAD) leads to induction of a reporter gene. (B) In the G-protein fusion method, the interaction between protein $\mathbf{X}$ and protein $Y$ occurs at the membrane, and sequesters $\mathbf{G} \beta \gamma$. Disruption of G-protein signaling leads to reduced transcription of a reporter gene and failure to undergo growth arrest. Drugs or mutations that disrupt binding of $X$ and $Y$ will restore G-protein signaling.

\section{Results}

Expression of syntaxin $\mathbf{l a}$ and $\mathbf{n S e c l - G} \gamma$. O ur central goal was to be able to monitor the binding of integral membrane proteins to their cytoplasmic protein targets in vivo. Our approach was to convert these interactions into a G-protein-mediated event. With fusion of a cytoplasmic binding partner to the G-protein $\gamma$-subunit, highaffinity interactions should disrupt G-protein-dependent changes in gene transcription and cell growth (Fig. 1).

As a first test of this approach, we expressed syntaxin la and a fusion of $\mathrm{nSecl}$ with $\mathrm{G} \gamma$ in yeast. We initially examined whether expression of syntaxin la or the nSecl-G $\gamma$ fusion alone would interfere with G-protein signaling, using the pheromone-dependent growth inhibition (halo) assay. A stel8s mutant ( $\mathrm{G} \gamma$-deficient) strain was transformed with plasmids expressing $n \operatorname{Sec} 1-\mathrm{G} \gamma$, syntaxin $1 \mathrm{a}$, or $\mathrm{G} \gamma$ (Ste18) alone. Cells were plated and then exposed to different amounts of synthetic $\alpha$-factor spotted onto filter disks. After $48 \mathrm{~h}$, cells expressing $\mathrm{nSec} 1-\mathrm{G} \gamma$ exhibited a clear zone of growth inhibition, comparable to that seen with $\mathrm{G} \gamma$ (Fig. 2A). These results indicate that attachment of a heterologous protein to the $\mathrm{N}$ terminus of $\mathrm{G} \gamma$ does not alter $\mathrm{G} \beta \gamma$ assembly or signaling in vivo. In contrast, coexpression of syntaxin $1 a$ and $n \operatorname{Sec} 1-G \gamma$ yielded considerably more turbid halos, indicating a potent inhibition of $G \beta \gamma$ signaling (Fig. 2A). Presumably $n S e c 1-G \gamma$ is capable of forming a functional dimer with $G \beta$, but one that binds preferentially to syntaxin $1 a$ and is therefore unable to activate a signaling pathway leading to growth arrest.

Table 1. nSec 1 mutations that disrupt binding to syntaxin 1a

$\begin{array}{ll}\text { S42F } & \text { Contact site } \\ \text { M 51K, K294R } & \text { Contact site, contact region } \\ \text { D112N } & \text { Contact region } \\ \text { I482T, K524M } & \text { Hinge region }\end{array}$

To confirm that binding is specific, we tested another isoform of syntaxin (syntaxin 4) that does not recognizenSec $1^{18}$. In this case, coexpression with $\mathrm{nSec} 1-\mathrm{G} \gamma$ yielded normal halos, comparable to $\mathrm{G} \gamma$ alone (data not shown). The expression and membraneassociation of nSecl-G $\gamma$ or syntaxin la was not altered under any of the conditions tested, as shown by immunoblotting (Fig. 2B).

To corroborate the results of the halo assay, and to providea more quantitative assessment of the change in pheromone signaling, we performed a reporter transcription assay (Fig. 2C). For these experiments we used the lacZ reporter gene under the control of the pheromone-inducible promoter from FUS1 (refs 19,20). As shown in Figure $2 \mathrm{C}$, expression of $\mathrm{nSec} 1-\mathrm{G} \gamma$ yielded $\beta$-galactosidase activities even higher than that seen with wild-type $G \gamma$. In contrast, coexpression of syntaxin 1 a with $n$ Secl-G $\gamma$ resulted in a marked decrease in the maximum level of induction, with no change in $\mathrm{EC}_{50}$ for pheromone induction. These data are consistent with the halo assay above, indicating that the $\mathrm{nSec} 1-\mathrm{G} \gamma$ can function in place of $\mathrm{G} \gamma$, but preferentially binds to syntaxin $1 a$.

Expression of FGFR3 and SNT-G $\gamma$ fusion. To determine if the G-protein fusion method can be used to monitor the interaction of other proteins, we tested a second pair consisting of FGFR3 and SNT-1. SNT-1 was selected because binding is independent of receptor activity ${ }^{21}$. FGFR3 was selected because it does not appear to be toxic to the host yeast cell, unlike several other tyrosine kinases (see below) ${ }^{22,23}$. Finally, FGFR3 is an attractive drug target, since the FGF signaling pathway is permanently activated in somecancer cells ${ }^{24,25}$.

As shown in Figure 3A, expression of the SNT-G $\gamma$ fusion yielded normal halos, comparable in size to $\mathrm{G} \gamma$ alone. However, cells coexpressing FGFR3 and SNT-G $\gamma$ exhibited moreturbid hal os, indicating a loss of $\mathrm{G}$-protein signaling. These results were corroborated by the transcription reporter assay (Fig. 3B). Again, expression of $\mathrm{G} \gamma$ or SNT-G $\gamma$ yielded nearly equivalent $\beta$-galactosidase responses, whereas co-expression of FGFR3 and SNT-G $\gamma$ yielded a marked decrease in the maximum level of induction. These results mirror those described above, using nSecl and syntaxin 1 a.

Genetic screen for $n$ Secl mutants that block binding to syntaxin 1a. A particular advantage of any yeast-based assay is the ability to carry out simple genetic screens on a large scale. Thus the approach described above could also be used to screen for mutations that modulate the interaction between any two protein-binding partners, even those not normally present in yeast. As an example, mutations in $\mathrm{nSecl}$ that disrupt binding to syntaxin la could easily be isolated, by screening for the reacquisition of pheromone responsiveness. A unique feature of this approach is that such mutants must not interfere with expression or overall folding of the protein, since the $\mathrm{G} \gamma \mathrm{moi}$ ety must retain the ability to bind $\mathrm{G} \beta$, even if $\mathrm{nSecl}$ activity is lost.

To isolate nSecl mutants that no longer bind syntaxin $1 a$, the $\mathrm{nSec} 1-\mathrm{G} \gamma$ fusion was amplified by polymerase chain reaction (PCR) using conditions designed to increase misincorporation of nucleotides ("error prone PCR"). The amplified products werethen co-transformed with the original plasmid, which had been digested so as to remove the entire $n S e c 1$ open reading frame. After transformation into yeast, recombination of the DNA fragments allows plasmid replication and cell growth on selective media. Resulting colonies were replica stamped to plates either with or without high concentrations of $\alpha$-factor. Rare $\alpha$-factor-sensitive colonies were then restreaked, and retested for pheromone sensitivity using the halo assay ( 58 colonies out of 15,000 screened). Finally, to confirm that the activity was conferred by the plasmid, episomal DNA was prepared from 13 colonies, amplified in Escherichia coli, and used to retransform the original stel8 $\Delta$ strain. After these manipulations, four candidate mutants were selected for sequencing (Table 1 ).

Sequencing of the $\mathrm{Sec} 1-\mathrm{G} \gamma$ mutants revealed single-site substitutions at position 42 (serine to phenylalanine, $\mathrm{nSec}{ }^{542 F}$ ) and 112 (aspartic acid to asparagine, $\mathrm{nSec} 1^{\mathrm{D} 112 \mathrm{~N}}$ ). The remaining mutants con- 
RES E AR C H ARTICLES
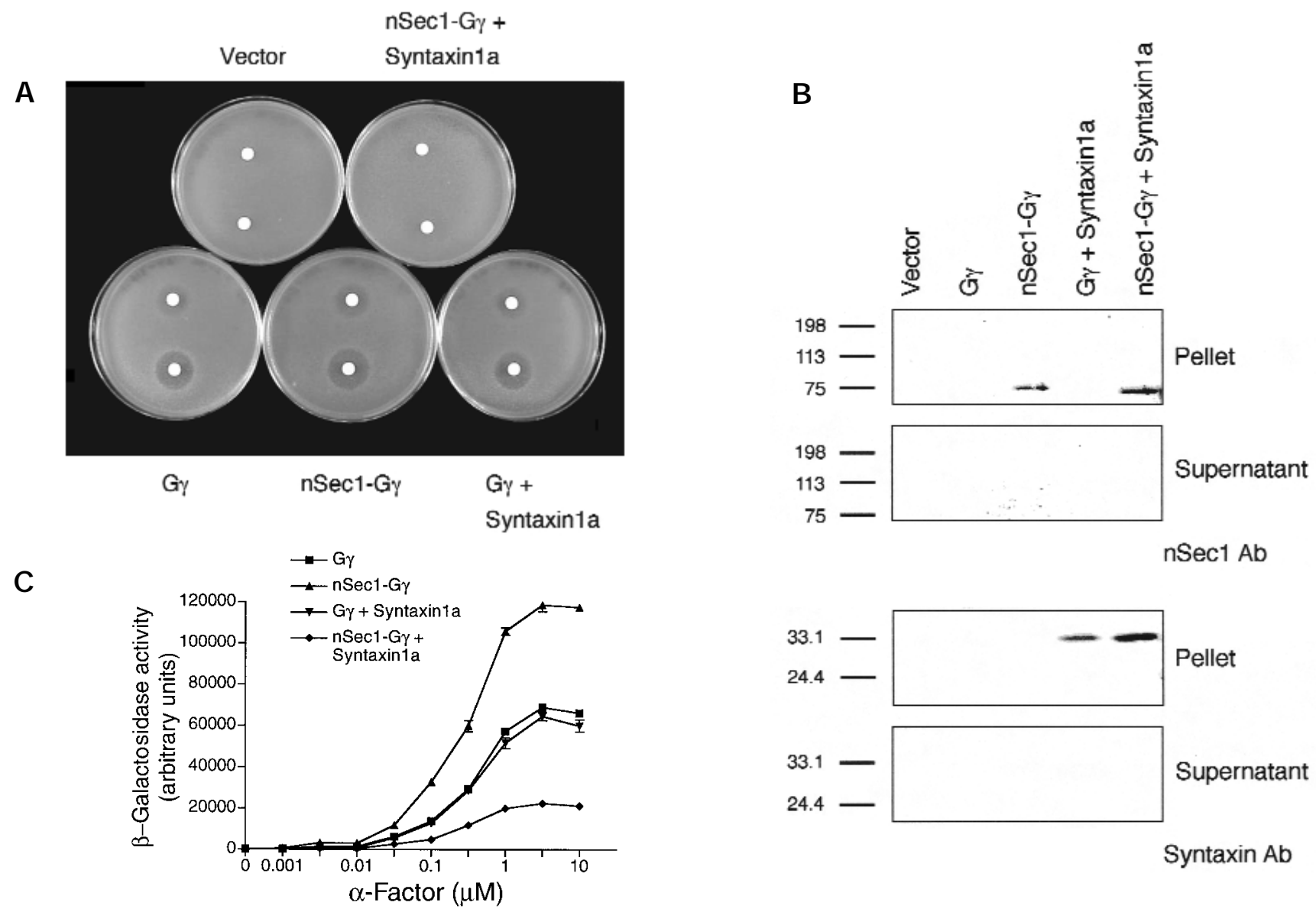

Figure 2. Detection of syntaxin la binding to $\mathrm{nSec}$. (A) Halo assay. $\mathbf{G} \gamma$-deficient cells (ste18 $\Delta$ mutant) were transformed with vectors containing no insert ("vector"), G $\gamma$, nSec 1-G $\gamma$ fusion, syntaxin 1a, or syntaxin 4 (not shown) as a negative control. Cells were plated and exposed to filter disks containing 20 or $48 \mu \mathrm{g} \alpha$-factor pheromone for $48 \mathrm{~h}$, and then photographed. (B) Immunoblot. To confirm expression of nSec 1 and syntaxin, cells were lysed, centrifuged to resolve membrane ("pellet") and cytosolic ("supernatant") fractions, and resolved by gel electrophoresis. Immunoblots were probed with antibodies to nSec1 (top panels) or syntaxin (bottom panels). Mobility of molecular weight standards is indicated. (C) Reporter transcription assay. Cells were treated with the indicated concentrations of $\alpha$-factor, and $\beta$-galactosidase activity was determined using a pheromone-responsive FUS1 promoter-lacZ reporter construct. Data shown are typical of two to five independent experiments performed in triplicate. Error bars, \pm s.e.

tained two substitutions, at 51 (methionine to lysine) and 294 (lysine to arginine) (nSec1 ${ }^{\mathrm{M} 51 \mathrm{~K}, \mathrm{~K} 294 \mathrm{R}}$ ) or at 482 (isoleucine to threonine) and 524 (lysine to methionine) (nSec1 ${ }^{1482 T}$, K524M $)$. All of these mutations can be rationalized in the context of the recently described crystal structure of the nSecl-syntaxin 1a complex ${ }^{13}$. This analysis revealed that $\mathrm{nSec} 1$ is composed of three domains, arranged in an arch that surrounds a portion of syntaxin 1a. Residues in the first and third domains form direct contacts with syntaxin $1 a$, and these contacts are largely polar or complementary in charge. Three of the mutants isolated in our screen alter a contact-site amino acid (S42F, M 51K) of the first domain or an amino acid within a contact region of the first (D112N) or third (K294R) domain. The remaining mutant affects residues distal to the contact interface, but that are part of a hinge region needed to form the arch that surrounds syntaxin la.

\section{Discussion}

All cell processes involve highly regulated protein-protein interactions. One particularly well-characterized example involves receptor- $G$ protein coupling, and the consequent dissociation and reassociation of G-protein subunits. Here, we describe an adaptation of this signaling apparatus that permits the detection of other protein-protein interactions in vivo. Our method is conceptually similar to the yeast two-hybrid method, where two binding partners are fused to the DNA-binding and transcriptional- activation domains of Gal4, respectively (Fig. 1A). In our method, one of the proteins is fused to the G-protein $\gamma$-subunit. Interaction between the two test proteins disrupts $G$ protein-dependent signaling, affecting such easily measured events as gene transcription and cell growth (Fig. 1B). In principle, other effects of G-protein activation could also be monitored, such as phosphorylation, protein translocation, cell morphogenesis, and fusion (mating). The availability of multiple signaling assays will likely reduce the incidence of false positives. In contrast, other well-known signaling pathways, such as that controlled by the small G-protein Ras, have not been nearly as well delineated in yeast ${ }^{5,6}$.

A major advantage of our method is that it can be used to detect protein-protein interactions at the plasma membrane. This is significant because about $40 \%$ of all proteins (including many important drug receptors) are thought to be anchored in the lipid bilayer, and are unlikely to enter thenucleus ${ }^{26}$. Thus, our approach could be used to identify drugs that bind receptors at the cell surface, and regulate the activity of sometarget protein inside the cell, through changes in receptor conformation. Some examples include receptor tyrosine kinases, ion channels, transporters, virus receptors, antigen receptors, and cell adhesion molecules.

A second advantage of our approach is that only one of the two binding partners needs to be expressed as a fusion protein. A protein in its native form is more likely to exhibit normal folding and ligand 


\section{RESEARCH ARTIC LES}

A

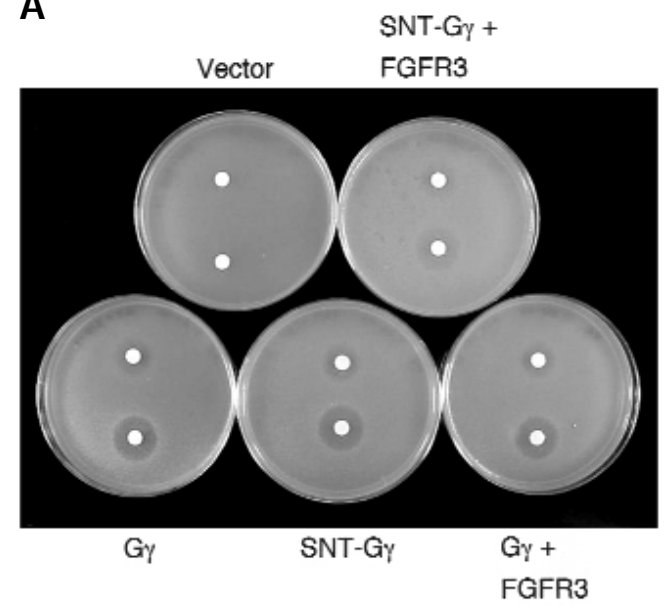

B

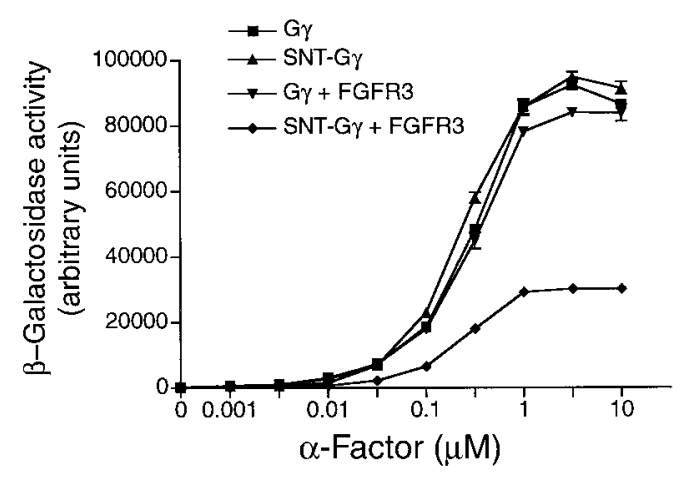

Figure 3. Detection of FGFR3 binding to SNT-1. (A) Halo assay. G $\gamma$-deficient cells (ste18 $\Delta$ mutant) were transformed with vectors containing no insert ("vector"), G $\gamma$, SNT-G $\gamma$ fusion, or FGFR3, as indicated. Cells were plated and exposed to filter disks containing 20 or $48 \mu \mathrm{g} \alpha$ factor pheromone for $48 \mathrm{~h}$, and then photographed. (B) Cells were treated with the indicated concentrations of $\alpha$-factor, and $\beta$-galactosidase activity was determined using a pheromoneresponsive FUSI promoter-lacZ reporter construct. Data shown are typical of two to five independent experiments performed in triplicate. Error bars, \pm s.e. binding properties. Even some native proteins cannot be functionally expressed in yeast, however. For instance, we have attempted to express the FGFR2 and HER-2/neu receptor without success. One alternative may be to express these proteins in cultured mammalian cells instead of in yeast.

Another goal for the future is to identify new binding partners for integral membrane proteins, including syntaxin $1 a$ and the FGFR3. The construction of CDNA libraries in standard two hybrid vectors has been extremely useful in this regard. A similar approach could be used to screen cDNAsfused to $\mathrm{G} \gamma$, or to screen for competitive inhibitors of known binding partners fused to $\mathrm{G} \gamma$.

Finally, we are interested in identifying new drugs that regulate FGFR3 or syntaxin la activity. Theability to find mutations that disrupt protein-protein interactions suggests that drugs with similar properties might be identified ${ }^{27}$. M oreover, a screen for mutants can usually be adapted into a screen for drugs, using the same readout. Syntaxin $1 \mathrm{la}$ is a known substrate for Clostridium botulinum toxin, and this agent is used therapeutically to treat conditions of overactive muscle contraction, such as spasticity in children with cerebral palsy, or spasms in patients with multiple sclerosis, stroke, or spinal cord injury ${ }^{12}$. Thus, the identification of new inhibitors of syntaxin la could lead to new drugs for the treatment of human disease.

In summary, we describe an approach to detect interaction between two proteins in vivo. We have demonstrated the utility of the method for two different protein-binding partners, and for carrying out genetic screens for mutants that are expressed normally but areno longer able to recognize their binding partner. This approach could be used to monitor the interactions between any two proteins in a cell, under various physiological and pharmacological conditions.

\section{Experimental protocol}

Strains, media, and plasmid construction. Standard methods for the growth, maintenance, and transformation of yeast and bacteria, and for the manipulation of DNA, were used throughout ${ }^{28}$. The yeast Saccharomyces cerevisiae strain used in this study was M HY6 (M ATa ura3-52 lys2-801 am ade2-101 ${ }^{\circ C}$ trp1-63 his3- 200 leu2-1 stel8:LEU2) (provided by Jeremy Thorner, University of California Berkeley).

STE18 was PCR amplified using a 5' oligonucleotide containing an ECORI site followed by sequence encoding MAHHHHHHASM (original start codon). The PCR product was ligated into the yeast expression vector pRS314-GAL (ref. 29) (ampr ${ }^{r}$ CEN/ARS, TRP1, GAL1/10 promoter) to yield pRS314-GAL-H 6-STE18. To prepare $G \gamma$ fusions, the coding sequence of rat nSec1 or human SNT-1 (with a C-terminal triple myc epitope tag, provided by M itchell Goldfarb, M t. Sinai School of M edicine) was PCR amplified and ligated into pRS314-GAL-H 6-STE18. Full-length rat syntax in la was PCR amplified and ligated into pRS316-ADH (ampr ${ }^{r}$, CEN/ARS, URA3, ADH 1 promoter and termination sequence) ${ }^{30}$. M ouse FGFR3, either wild type (data not shown) or containing the TDII-type mutation ${ }^{31}$, was PCR amplified, and then ligated by co-transformation and homologous recombination into vector pRS423-GAL (ampr, $2 \mu$, HIS3, GAL1/10 promoter, CYC1 terminator) or pRS426-GAL ( $\mathrm{amp} \mathrm{p}^{r}, 2 \mu, \mathrm{URA} 3, \mathrm{GAL} 1 / 10$ promoter, CYC1 terminator).

Mutagenesis of the GAL-nSec1-H6-STE18 cassette was carried out using "error-prone PCR," as described ${ }^{32}$. The amplified products were recombined with pRS314-GAL-nSec1-H 6-STE18, which had been digested with EcoRI so as to remove the entire nSecl open reading frame, by cotransformation and nutritional selection. Resulting colonies were replica stamped to plates either with or without $\geq 40 \mu \mathrm{M}$ of $\alpha$-factor. Rare $\alpha$-factor-sensitive colonies were then restreaked, and retested for pheromone sensitivity using the halo assay. Plasmid DNA was prepared from each colony, amplified in E. coli, and used to retransform the original stel $8 \Delta$ strain, and retested by the halo assay.

Cell disruption, membrane fractionation, and immunoblot analysis. For preparation of whole-cell lysates, cell pellets were resuspended in 1× sample buffer for sodium dodecyl sulfate-polyacrylamide gel electrophoresis (SDS-PAGE), boiled for $10 \mathrm{~min}$, and subjected to glass bead vortex homogenization for $2 \mathrm{~min}$. Fractionated cell lysates were prepared as described ${ }^{30}$. Protein extracts were resolved by $8 \%$ or $12 \%$ SDS-PAGE and transferred to nitrocellulose. Blots were probed with antibodies against nSecl (supplied by Pietro DeCamilli, YaleUniversity) or syntaxin (supplied by Colin Barnstable, Yale University). Blots were also performed using antibodies against syntaxin 4 (Chemicon International, Temicula, CA), FGFR3 (C-15; Santa Cruz Biotechnology Inc., Santa Cruz, CA), or FRS2 (H-91; Santa Cruz Biotechnology Inc.) (data not shown). SNT-1 is homologous to mouse FRS2. Antibody detection was achieved using horseradish peroxidase-conjugated goat anti-mouse IgG (Bio-Rad Laboratories, Hercules, CA) or goat antirabbit IgG (Bio-Rad Laboratories) and colorimetric ${ }^{28}$ or chemiluminescence detection (New England Nuclear, Boston, MA; Pierce Chemical Co., Rockford, IL) according to the manufacturer's instructions.

Pheromone response assays. The pheromone-dependent growth inhibition assay (halo assay) was performed as described ${ }^{30}$.

For pheromone-dependent reporter-transcription assays ${ }^{19}$, mid-log phase cells were aliquoted $(90 \mu \mathrm{l})$ to a 96-well plate, and mixed with $10 \mu \mathrm{l}$ of $\alpha$-factor for $90 \mathrm{~min}$, in triplicate. $\beta$-galactosidase activity was measured by adding $20 \mu \mathrm{l}$ of a freshly prepared solution of $83 \mu \mathrm{M}$ fluorescein di- $\beta$-D-galactopyranoside ( $10 \mathrm{mM}$ stock in dimethyl sulfoxide; Molecular Probes, Eugene, OR), 137.5 mM PIPES pH 7.2, 2.5\% Triton X-100, and incubating for $90 \mathrm{~min}$ at $37^{\circ} \mathrm{C}$. The reaction was stopped by the addition of $20 \mu \mathrm{l}$ $1 \mathrm{M} \mathrm{Na}_{2} \mathrm{CO}_{3}$, and the resulting fluorescence activity was measured at $485 \mathrm{~nm}$ excitation, $530 \mathrm{~nm}$ emission. Because of differences in instrument calibration, sample number, and the like, data from each experiment are presented as arbitrary units rather than absolute values.

\section{Acknowledgments}

This work was supported by NIH grants AR44906 (X-Y.F.), GM 55316 and GM 59167 and pilot funds from the YaleCancer Center (to H.G.D.). K.N.E. is an NIH predoctoral trainee(T32-GM 07324). H.G.D. is an established investigator of theAmerican Heart Association. 
RES E AR C H ARTICLES

1. Fields, S. \& Song, O. A novel genetic system to detect protein-protein interactions. Nature 340, 245-246 (1989).

2. Bai, C. \& Elledge, S. J. Gene identification using the yeast two-hybrid system. Methods Enzymol. 273, 331-347 (1996).

3. J ohnsson, N. \& Varshavsky, A. Split ubiquitin as a sensor of protein interactions in vivo. Proc Natl Acad Sci USA 91, 10340-10344 (1994).

4. Stagljar, I., Korostensky, C., J ohnsson, N. \& te Heesen, S. A genetic system based on split-ubiquitin for the analysis of interactions between membrane proteins in vivo. Proc Natl Acad Sci USA 95, 5187-5192 (1998).

5. Aronheim, A., Zandi, E., Hennemann, H., Elledge, S. J . \& Karin, M. Isolation of an AP-1 repressor by a novel method for detecting protein-protein interactions. Mol. Cell Biol. 17, 3094-3102 (1997).

6. Broder, Y.C., Katz, S. \& Aronheim, A. The ras recruitment system, a novel approach to the study of protein-protein interactions. Curr Biol. 8, 1121-1124 (1998).

7. Pelletier, J . N., Campbell-Valois, F.X. \& Michnick, S. W. Oligomerization domaindirected reassembly of active dihydrofolate reductase from rationally designed fragments. Proc. Natl. Acad. Sci. USA 95, 12141-12146 (1998).

8. Remy, I., Wilson, I.A. \& Michnick, S.W. Erythropoietin receptor activation by a ligand-induced conformation change. Science 283, 990-993 (1999).

9. Rossi, F.M., Blakely, B.T. \& Blau, H.M. Interaction blues: protein interactions monitored in live mammalian cells by beta-galactosidase complementation. Trends Cell. Biol. 10, 119-122 (2000).

10. Blakely, B.T. et al. Epidermal growth factor receptor dimerization monitored in live cells. Nat. Biotechnol. 18, 218-222 (2000).

11. Dohlman, H.G., Song, J., Apanovitch, D.M., DiBello, P.R. \& Gillen, K.M. Regulation of $\mathrm{G}$ protein signalling in yeast. Semin. Cell. Dev. Biol. 9, 135-141 (1998).

12. Hallett, M. One man's poison-clinical applications of botulinum toxin. N. Engl. J. Med. 341, 118-120 (1999)

13. Misura, K.M., Scheller, R.H. \& Weis, W.I. Three-dimensional structure of the neuronal-Sec 1-syntaxin 1a complex. Nature 404, 355-362 (2000).

14. Burke, D., Wilkes, D., Blundell, T.L. \& Malcolm, S. Fibroblast growth factor receptors: lessons from the genes. Trends Biochem. Sci. 23, 59-62 (1998).

15. McKeehan, W.L., Wang, F. \& Kan, M. The heparan sulfate-fibroblast growth factor family: diversity of structure and function. Prog. Nucleic Acid Res. Mol. Biol. 59, 135-176 (1998).

16. Raffioni, S., Thomas, D., Foehr, E.D., Thompson, L.M. \& Bradshaw, R.A. Comparison of the intracellular signaling responses by three chimeric fibroblast growth factor receptors in PC12 cells. Proc. Natl. Acad. Sci. USA 96, 7178-7183 (1999).
17. Kanai, M., Goke, M., Tsunekawa, S. \& Podolsky, D.K. Signal transduction pathway of human fibroblast growth factor receptor 3 . Identification of a novel 66kDa phosphoprotein. J. Biol. Chem. 272, 6621-6628 (1997).

18. Pevsner, J., Hsu, S.C. \& Scheller, R.H. n-Sec1: a neural-specific syntaxin-binding protein. Proc. Natl. Acad. Sci. USA 91, 1445-1449 (1994).

19. Trueheart, J ., Boeke, J.D. \& Fink, G.R. Two genes required for cell fusion during yeast conjugation: evidence for a pheromone-induced surface protein. Mol. Cell Biol. 7, 2316-2328 (1987).

20. Sprague, G. F., J r. Assay of yeast mating reaction. Methods Enzymol. 194, 77-93 (1991).

21. Xu, H., Lee, K.W. \& Goldfarb, M. Novel recognition motif on fibroblast growth factor receptor mediates direct association and activation of SNT adapter proteins. J. Biol. Chem.. 273, 17987-17990 (1998).

22. Kornbluth, S., J ove, R. \& Hanafusa, H. Characterization of avian and viral p60src proteins expressed in yeast. Proc. Natl. Acad. Sci. USA 84, 4455-4459 (1987).

23. Walkenhorst, J ., Goga, A., Witte, O.N. \& Superti-Furga, G. Analysis of human cAbl tyrosine kinase activity and regulation in S. pombe. Oncogene $\mathbf{1 2}$ 1513-1520 (1996)

24. Chesi, M. et al. Frequent translocation $\mathrm{t}(4 ; 14)(\mathrm{p} 16.3 ; \mathrm{q} 32.3)$ in multiple myeloma is associated with increased expression and activating mutations of fibroblast growth factor receptor 3. Nat. Genet. 16, 260-264 (1997).

25. Cappellen, $D$, et al. Frequent activating mutations of FGFR3 in human bladder and cervix carcinomas. Nat. Genet. 23, 18-20 (1999).

26. Goffeau, A. et al. Life with 6000 genes. Science 274, 546, 563-547 (1996).

27. Gibbs, J.B. \& Oliff, A. Pharmaceutical research in molecular oncology. Cell 79, 193-198 (1994).

28. Ausubel, F.M., et al. (eds). Current protocols in molecular biology. (Greene Pub. Associates and Wiley-Interscience, New York, NY; 1987).

29. Sikorski, R.S. \& Hieter, P. A system of shuttle vectors and yeast host strains designed for efficient manipulation of DNA in Saccharomyces cerevisiae. Genetics 122, 19-27 (1989).

30. Song, J., Hirschman, J., Gunn, K. \& Dohlman, H.G. Regulation of membrane and subunit interactions by $\mathrm{N}$-myristoylation of a $\mathrm{G}$ protein alpha subunit in yeast. J . Biol. Chem. 271, 20273-20283 (1996).

31. Su, W. C. et al. Activation of Stat1 by mutant fibroblast growth-factor receptor in thanatophoric dysplasia type II dwarfism. Nature 386, 288-292 (1997).

32. Staples, R.R. \& Dieckmann, C.L. Generation of temperature-sensitive cbp1 strains of Saccharomyces cerevisiae by PCR mutagenesis and in vivo recombination: characteristics of the mutant strains imply that $C B P 1$ is involved in stabilization and processing of cytochrome b pre-mRNA. Genetics 135, 981-991 (1993). 